\title{
Coordenação nas Atividades Colaborativas em Ambientes de Aprendizagem - Uma Avaliação na Implementação de Arquiteturas Pedagógicas
}

\author{
Ramon Rosa Maia Vieira Júnior - PPGI-UFES - rmaia@inf.ufes.br \\ Otávio Lube dos Santos - PPGI-UFES - olube@inf.ufes.br \\ Jadson do Prado Rafalski - PPGI-UFES - jrafalski@inf.ufes.br \\ Everton Moschen Bada - PPGI-UFES - ebada@inf.ufes.br \\ Halysson Freitas Alves da Silva - PPGI-UFES - hfreitas@ inf.ufes.br \\ Crediné Silva de Menezes - PPGI-UFES - credine@inf.ufes.br
}

\begin{abstract}
Resumo. Motivados pela crescente oferta de Ambientes Virtuais de Aprendizagem realizamos um estudo na implementação de arquiteturas pedagógicas, sob a ótica da articulação e coordenação em atividades colaborativas, utilizando como referência o modelo 3C de Colaboração. Através destas Arquiteturas Pedagógicas, identificamos um conjunto de requisitos para redução do esforço cognitivo, causados pelos conflitos, na cooperação e apresentamos como resultados lacunas tecnológicas, no suporte das mesmas, em ambientes virtuais selecionados.
\end{abstract}

Palavras-chave: arquiteturas pedagógicas, ambientes virtuais de aprendizagem, coordenação, $\csc$.

\section{Coordination on the Collaborative Activities in Learning Environments - An Assessment on the Implementation of Pedagogical Architectures}

Abstract. Motivated by the availability increasing of learning management systems we have studied the implementation of pedagogical architectures, from the perspective of articulation and coordination of collaborative activities, using as reference the $3 C$ Model of Collaboration. Through these pedagogical architectures, we identified a set of requirements to reduce the cognitive effort, produced by possible conflicts, in cooperation and present as results technology gaps in support of them, in virtual environments selected.

Keywords: pedagogical architectures, virtual learning environments, coordination, $\csc$.

\section{Introdução}

O movimento em prol de pedagogias abertas vem oferecendo cada vez mais novas abordagens para construção de conhecimento através de redes de colaboração. Nesta perspectiva, Carvalho et. al. (2005) afirma que:

"[...] propostas pedagógicas concebidas para mediação da aprendizagem buscarão dar suporte a novas concepções educacionais, caracterizadas por deslocamento das concepções hierárquicas e disciplinares em direção a uma concepção de conhecimento interdisciplinar". Concepções desta natureza têm presente modelos de formação de professores que privilegiam modos de saber alimentados e potencializados na tessitura composta pela formação aberta apoiada por "rede de relações". (Carvalho et. al., 2005).

O uso e a produção de ambientes virtuais para a realização de atividades colaborativas conduziram muitas indagações, entre as quais se destacam as seguintes: (i) Os ambientes virtuais para o ensino e aprendizagem estão capacitados para apoiar a 
inovação pedagógica? (ii) As arquiteturas pedagógicas podem ser adotadas como estratégias e implementadas nos ambientes virtuais atuais? (iii) Quais são os aspectos críticos das atividades colaborativas que precisam ser endereçados pelos ambientes virtuais?

Neste trabalho, partindo destes questionamentos, avaliamos um conjunto de ambientes virtuais, sob uma perspectiva crítica da colaboração, na implementação de Arquiteturas Pedagógicas.

\section{Arquiteturas Pedagógicas}

O uso das tecnologias da informação e da comunicação, potencializados pelo surgimento da web 2.0 e com a web 3.0 já em andamento, vem demandando novos estudos que busquem dar aos educadores uma oportunidade para discutirem sobre o aporte das tecnologias no processo educacional. Busca-se inverter a lógica corrente onde as ferramentas surgem e os educadores tentam encontrar usos adequados dessas tecnologias para modernizarem seus cursos.

É dentro desta perspectiva que Carvalho et al. (2005) conceberam o conceito de Arquiteturas Pedagógicas, buscando pensar o uso das tecnologias sintonizado com as novas propostas e vice-versa, como podemos observar no trecho:

"Alteram-se as perspectivas de tempo e espaço para a aprendizagem, porque o conhecimento tem como ponto de partida arquiteturas plásticas. Estas se moldam aos ritmos impostos pelo sujeito que aprende”. (Carvalho et. al., 2005).

Nesta concepção, Carvalho et al. (2005) define:

“Arquiteturas pedagógicas (APS) são estruturas de aprendizagem realizadas a partir da confluência de diferentes componentes: abordagem pedagógica, software, internet, inteligência artificial, educação a distância, concepção de tempo e espaço. O caráter destas arquiteturas pedagógicas é pensar a aprendizagem como um trabalho artesanal, construído na vivência de experiências e na demanda de ação, interação e meta-reflexão do sujeito sobre os fatos, os objetos e o meio ambiente sócio-ecológico. Seus pressupostos curriculares compreendem pedagogias abertas capazes de acolher didáticas flexíveis, maleáveis, adaptáveis a diferentes enfoques temáticos.”. (Carvalho et. al., 2005).

Onde fica notório a preocupação com o uso da tecnologia sintonizado com novas propostas, que se originam nas novas possibilidades de comunicação e cooperação viabilizadas pelas redes telemáticas, que rompem as barreiras de tempo e espaço, permitindo ao educador o compartilhamento de idéias com seus pares e estudantes, a qualquer hora e em qualquer lugar.

Preocupação que é reforçada no trecho a seguir onde se busca enfatizar a metamorfose das interações, sugerindo o abandono das hierarquias em favorecimento das relações interdisciplinares.

As arquiteturas pedagógicas configuram-se como releituras de abordagens pedagógicas ao realizarem a intersecção entre projeto educativo e o suporte telemático. Buscam traduzir em situações de aprendizagem propostas pedagógicas concebidas para a mediação da aprendizagem, caracterizadas por deslocamento das concepções hierárquicas e disciplinares de ensino, na direção de uma concepção do conhecimento interdisciplinar e do modelo de formação de professores como rede de relações. (Carvalho et al., 2005).

\section{Ambientes Virtuais de Aprendizagem e o Suporte Telemático}

Segundo Pessoa e Menezes (2003), a rede de computadores pode: i) mudar a maneira 
com que aprendizes e professores interagem; ii) aumentar as oportunidades para aprendizagem colaborativa; iii) facilitar as discussões; iv) deslocar o estudo de um processo individual em direção à aprendizagem social e participativa.

No entanto, Gómez (2001) citado por Carvalho (2005) entende que a incorporação das tecnologias nos "desenhos" globais de teleformação não garante, por si só, a efetividade dos resultados, devendo estar sustentada por uma teoria de aprendizagem que justifique esse "desenho" e o delimite.

Um Ambiente Virtual de Aprendizagem (AVA) tem por finalidade, em sua concepção, oferecer um suporte tecnológico para apoiar as práticas de ensino-aprendizagem provendo entre outros, recursos para facilitar a interação dos participantes. Entretanto, muitos desses ambientes, conforme Pessoa e Menezes (2003), Monteiro et al. (2005), Menezes et al. (2008), requerem adaptação das práticas pedagógicas aos AVAs e não o contrário, o que reflete na redução da inovação pedagógica, quando implementados nestes ambientes. Ou ainda, um aumento do esforço cognitivo na apropriação de novas ferramentas para suprir as necessidades de um grupo de aprendizes, quando estes ambientes não apóiam suas necessidades de trabalho.

Assim, as demandas de novas arquiteturas pedagógicas resultam em modificações que implicam em inadequações com as demais. Percebe-se uma alta demanda e um esforço de produção atrelados à Engenharia de Software para o desenvolvimento de uma solução final limitada, capaz de atender apenas necessidades imediatas.

\section{Modelo 3C de Colaboração}

Os trabalhos de pesquisa em CSCW (Computer Supported Cooperative Work), oferecem os fundamentos necessários para apoiar nossa avaliação sobre sistemas colaborativos. Assim como outros sistemas colaborativos online, os AVAs permitem interação remota entre os participantes, em um ambiente compartilhado, reduzindo os conflitos e facilitando a interação, no que se refere aos aspectos de tempo e espaço.

Os sistemas colaborativos são definidos por Ellis (1991) como: groupwares, isto é, sistemas baseado em computador que suporta grupos de pessoas comprometidas em uma tarefa (objetivo) comum e fornecem uma interface para um ambiente compartilhado. Ellis (1991) ainda complementa definindo o objetivo destes sistemas como: assistir grupos na comunicação, na coordenação e na colaboração (corresponde a cooperação no modelo 3C). Sob os mesmos aspectos, a colaboração é a inter- e intrarelação entre comunicação, coordenação e cooperação, segundo Fuks et al. (2007) e Borghoff e Schlichter (2000).

Segundo o modelo 3C, apresentado em Fuks et al. (2007), reproduzido na Figura 1, a negociação e os compromissos acontecem em decorrência da comunicação entre os participantes (colaboradores). A coordenação gerencia os conflitos, organiza as tarefas com objetivo de prevenir a perda de comunicação e de esforço da cooperação. A cooperação é o trabalho conjunto de um grupo de indivíduos sob um espaço compartilhado, objetivando a execução de tarefas. Durante a cooperação acontece a criação e/ou a manipulação de objetos/recursos (artefatos). 


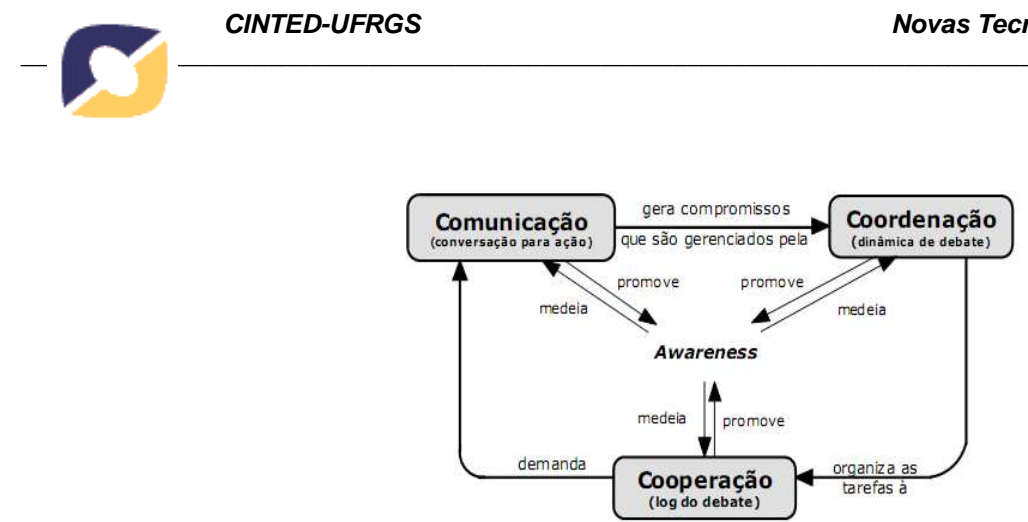

Figura 1: Modelo 3C de colaboração. (Fuks et al., 2007).

Geralmente, as atividades colaborativas seguem, ou definem, as regras de interação sob duas formas: utilizando o protocolo social (Fuks et al., 2007) ou através de recursos funcionais do sistema. Para que as atividades cooperativas ocorram de acordo com um determinado protocolo de interações é necessário que ocorra um planejamento, considerando os elementos (atores, artefatos, tarefas e ambiente) da atividade.

Antes de a colaboração iniciar ocorre uma fase de planejamento: articulação. Esta consiste em todas as tarefas envolvidas na preparação do ambiente, agendamento, monitoramento e coordenação de todas as etapas necessárias para completar as atividades (Schmidt, Bannon, 1992). Considera-se ainda, como fase do processo de articulação atividades como: identificação dos objetivos, o mapeamento destes objetivos em tarefas, a seleção dos participantes e a delegação das tarefas.

Trabalhar em grupo traz motivação para o membro, pois seu trabalho estará sendo observado, comentado e avaliado por outros participantes. Entretanto, trabalhar cooperativamente demanda esforço adicional para a coordenação de seus membros. Sem a coordenação é possível que parte dos esforços de comunicação não sejam aproveitada na cooperação, isto é, para que o grupo possa trabalhar em conjunto de forma harmoniosa, é necessário que os compromissos assumidos nas conversações sejam gerenciados para evitar conflitos interpessoais, que possam prejudicar o grupo. Desta forma, sem a coordenação, os colaboradores poderão engajar em atividades conflitantes ou repetitivas (Schmidt, Bannon, 1992).

\section{Avaliação}

Nosso trabalho tem como objetivo avaliar os AVAs, sob análise do suporte tecnológico, na implementação das APs para o atendimento dos requisitos das atividades de articulação e coordenação das atividades colaborativas. Isto se realizará com base em algumas arquiteturas, onde os aspectos mais relevantes serão elicitados e em AVAs específicos, escolhidos a partir de algumas características.

\subsection{As Arquiteturas Pedagógicas selecionadas}

Selecionamos algumas APs encontradas na literatura para apoiar este estudo. Abaixo, uma rápida descrição sobre cada uma:

Construindo conceituações: $O$ que se pretende promover com esta arquitetura é que indivíduos em processo de compreensão de um determinado micromundo elaborem suas conceituações apoiados por uma "rede de interações" (Nevado et al., 2009). Nesta arquitetura o mediador apresenta algumas teses sobre o micromundo a ser estudado e cada participante apresenta seu posicionamento inicial sobre elas. Este posicionamento será em seguida refinado com base em uma rede de trocas. No momento seguinte, todos os participantes se envolvem do processo de revisão, revisando e sendo revisado. Nestas interações o aluno aprenderá a argumentar sobre determinado micromundo. No 
desfecho o individuo tem a possibilidade de reapresentar a sua argumentação, evidenciando assim as aprendizagens construídas.

Diário Virtual: Essa AP tem como objetivo prover um canal diferenciado de comunicações entre o estudante e professor. Com o diário virtual o professor consegue acompanhar o entendimento sobre o assunto estudado, e também entende a evolução do conhecimento do aluno. Com isso, caso o professor identifique que o estudante está com um conceito errado sobre determinado assunto, ele conseguirá determinar em qual momento da aprendizagem isto ocorreu, e então propor alguma leitura/atividade ao estudante para que ele sozinho consiga perceber o equivoco e consertar o conceito (Serres e Basso, 2009).

Projeto de Aprendizagem (PA): O objetivo é o desenvolvimento de um processo de aprendizagem que alcance a construção de novos conhecimentos, em que o aprendiz possa sistematizar informações ampliando sua rede de significações e reestruturar o raciocínio lógico sobre os novos significados enquanto elabora a síntese de respostas descritivas e explicativas para sua curiosidade (Fagundes et al., 2006). Tudo começa com um levantamento de perguntas de interesse dos participantes, originadas em suas curiosidades. As perguntas são agrupadas por aproximação o que dá então suporte aos grupos de trabalho. As curiosidades são a seguir exploradas com respeito ao conhecimento prévio, manifestado no PA através das certezas provisórias e das dúvidas temporárias. O processo cooperativo consiste no esclarecimento dessas dúvidas e na validação da certezas, através da consulta a fontes, da experimentação, da simulação, da coleta de dados, da observação, etc. O desfecho é a elaboração de respostas à pergunta de investigação através do uso de artefatos e eventos utilizando diferentes linguagens, mídias e suportes.

Controvérsia Acadêmica: A Controvérsia Acadêmica é um método de aprendizagem cooperativa cujo objetivo é configurar conflitos acadêmicos como atividade altamente construtiva. De forma simples, pode-se dizer que a Controvérsia Acadêmica existe quando uma ideia, informação, conclusão, teoria ou opinião de um estudante é incompatível com a de outro, e os dois procuram chegar a um consenso (Johnson et al., 1998).

Júri Simulado: Esta AP busca contribuir para a construção do conhecimento por meio do desenvolvimento da argumentação, das possibilidades de cooperação, criatividade e ludicidade. Existem 5 papéis diferentes: réu, juiz, defesa, acusação e os jurados onde todos são bem definidos e não pode ocorrer mudança dos mesmos. O Júri Simulado é um evento que por si só propõe a interatividade, pois os grupos de ataque e defesa são constituídos em forma de fórum, no qual cada aluno pode acompanhar os argumentos dos dois grupos e se posicionar (Real e Menezes, 2007).

\subsection{Os Ambientes Virtuais de Aprendizagem considerados}

Realizamos uma seleção dos AVAs para a nossa avaliação e alguns critérios influenciaram na seleção. Popularidade, estabilidade, disponibilidade, tipo de licença de software (software livre), foram os principais critérios. Também utilizamos como referência estudos recentes sobre LMS (Learning Management System), como Liu et al. (2009), para nos apoiar na seleção dos softwares.

Os AVA's escolhidos neste artigo para a realização da avaliação das APs, foram: Socrates (Nascimento et al., 2008), Amadeus (Gomes e Tedesco, 2002), Moodle (Moodle, 2011), Atutor (Lengyel et al., 2006), Dokeos (Dokeos, 2011), Claroline (Liu et al., 2009), Chamilo (Maes, 2010), Eureka (Eureka, 2011). 


\subsection{Elicitação dos Requisitos}

Conforme mencionado na seção anterior, as atividades de articulação e coordenação da colaboração possuem a responsabilidade de organizar o grupo, para evitar que esforços na cooperação e comunicação sejam perdidos, e as tarefas para que sejam realizadas na ordem correta, no tempo correto e atendendo as restrições e os objetivos.

Tabela 1: Requisitos das Arquiteturas Pedagógicas.

\begin{tabular}{|c|c|c|c|c|c|c|}
\hline & Requisitos & \begin{tabular}{|c|} 
Construindo \\
Conceituações
\end{tabular} & $\begin{array}{c}\text { Projeto de } \\
\text { Aprendizagem }\end{array}$ & $\begin{array}{c}\text { Controvérsia } \\
\text { Acadêmica }\end{array}$ & $\begin{array}{c}\text { Juri } \\
\text { Simulado }\end{array}$ & $\begin{array}{l}\text { Diário } \\
\text { Virtual }\end{array}$ \\
\hline \multirow{10}{*}{ 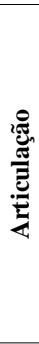 } & C.E.R. & $\mathrm{X}$ & $\mathrm{X}$ & $\mathrm{X}$ & $\mathrm{X}$ & $\mathrm{X}$ \\
\hline & \begin{tabular}{|l|} 
Convidar usuários \\
\end{tabular} & $\mathrm{X}$ & $\mathrm{X}$ & $\mathrm{X}$ & $\mathrm{X}$ & $\mathrm{X}$ \\
\hline & Determinação de Papeis & $\mathrm{X}$ & $\mathrm{X}$ & $\mathrm{X}$ & $\mathrm{X}$ & $\mathrm{X}$ \\
\hline & Dependência de Atividades & $X$ & $\mathrm{X}$ & $\mathrm{X}$ & $\mathrm{X}$ & $\mathrm{X}$ \\
\hline & Restrição de Interações* & $\mathrm{X}$ & & & & \\
\hline & Perfis Dinâmicos & $X$ & $\mathrm{X}$ & $\mathrm{X}$ & $\mathrm{X}$ & $\mathrm{X}$ \\
\hline & Definir Permissões de Leitura & $\mathrm{X}$ & & & $\mathrm{X}$ & \\
\hline & Definir Prazo de Leitura & $\mathrm{X}$ & & & $\mathrm{X}$ & \\
\hline & Definir Permissões de Escrita & $\mathrm{X}$ & $\mathrm{X}$ & $\mathrm{X}$ & $\mathrm{X}$ & $\mathrm{X}$ \\
\hline & Definir Prazo de Escrita & $\mathrm{X}$ & & $\mathrm{X}$ & $\mathrm{X}$ & $\mathrm{X}$ \\
\hline \multirow{5}{*}{ 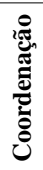 } & Multiplicidade de Papeis & $\mathrm{X}$ & & & & $\mathrm{X}$ \\
\hline & Redefinir Permissões de Leitura & $\mathrm{X}$ & & & $\mathrm{X}$ & \\
\hline & Redefinir Prazo de Leitura & $\mathrm{X}$ & & & $\mathrm{X}$ & \\
\hline & Redefinir Permissões de Escrita & $\mathrm{X}$ & $\mathrm{X}$ & $\mathrm{X}$ & $\mathrm{X}$ & $\mathrm{X}$ \\
\hline & Redefinir Prazo de Escrita & $\mathrm{X}$ & & $\mathrm{X}$ & $\mathrm{X}$ & $\mathrm{X}$ \\
\hline
\end{tabular}

Conforme nossa metodologia de avaliação, tentamos realizar uma implementação de uma instância de cada AP nos AVAs selecionados. Realizamos a implementação avaliando a conformidade dos AVAs com os requisitos da articulação e coordenação das atividades colaborativas.

\subsubsection{Requisitos de Articulação}

Os requisitos analisados, apresentados na Tabela 1, foram categorizados entre requisitos de articulação e coordenação. Criação de ambientes, convidar usuários, determinar papéis, dependência de atividades, restrição de interações, perfis dinâmicos, permissões de leitura e escrita e prazos de leitura e escrita são considerados requisitos de articulação. Embora alguns destes requisitos possam também ser considerados como requisitos de coordenação decidimos por esta categorização, pela sua frequente execução na etapa de articulação.

Criação de espaço reservado (C.E.R.) é o requisito funcional para estabelecer um espaço reservado dentro do ambiente virtual, capaz de satisfazer a criação de um espaço compartilhado para acesso dos participantes. Um dos propósitos deste espaço poderia ser alocação da implementação de uma AP, dentro do AVA. Convidar usuários é o requisito que permite o ambiente enviar um convite (ou um aviso) para um indivíduo, futuro participante, para fins de cadastro no ambiente.

Antes de prosseguirmos sobre os outros requisitos, é necessário falarmos sobre três elementos: Usuário, Perfil e Papel. Usuário é um participante (indivíduo registrado) presente no ambiente. Perfil é um apelido para representar um conjunto de responsabilidades, tendo como exemplo: mediador, aluno, revisor, entre outros. Papel é associação entre um usuário e um Perfil, isto é, delegar ao usuário X o Perfil Y. Desta forma, o Perfil, que poderá possuir um conjunto de permissões, estará associado à um usuário quando um Papel for criado.

A interação entre os participantes, por sua maioria, é realizada através da troca de mensagens, mapeadas pelas APs e denominadas, neste trabalho, como atividades.

Determinar Papéis é a capacidade de um ambiente realizar de forma dinâmica a 
atribuições de Perfis aos usuários. Dependência de Atividades é um requisito que estabelece a possibilidade de uma atividade estar dependente de uma outra, ou seja Atividade $\mathrm{Y}$ somente poderá ser iniciada após a atividade $\mathrm{X}$ finalizar. Restrição de Interações é o requisito que define a possibilidade do ambiente limitar a quantidade de interações de um Perfil, em uma atividade de cooperação ou comunicação, através do ambiente, ou seja, um aluno poder realizar somente $\mathrm{X}$ argumentações (postagens) em um Debate no ambiente. Caso o ambiente permita a dependência de atividades $e$ restrições de interações proverá uma maior controle e flexibilidade na gestão de atividades. Perfis Dinâmicos é a capacidade de um ambiente apoiar a criação de novos perfis, além dos Padrões, que o ambiente possivelmente oferece.

Em AVAs é muito comum possuir perfis padrões do ambiente: Aluno, Professor e Tutor. No entanto, a possibilidade de criamos novos perfis possibilita um avanço na flexibilidade no agrupamento de usuários e ainda oferece uma melhor identificação na distribuição das responsabilidades para um grupo de participantes. Mediador, Revisor, Jurado são alguns exemplos de perfis utilizados nas APs.

Definir permissão de leitura e escrita é um requisito que possibilita atribuir uma permissão, de leitura e/ou escrita, a um Perfil segundo uma determinada atividade. No planejamento de uma atividade em grupo mais complexa, como algumas APs propõem, algumas informações (postagens) ou atividades devem ser privadas e outras públicas. Desta forma, o acesso a estas informações devem ser restritos. A permissão pode variar de perfil à perfil, no entanto o ambiente deve prover recursos para gerenciar estes acessos. Esta permissão pode , se necessário, estabelecer restrições cronológicas, isto é, determinar um intervalo de tempo para o acesso, ou seja, Definir Prazo de Leitura e Escrita.

\subsubsection{Requisitos de Coordenação}

Enquanto os requisitos de articulação são responsáveis por preparar o ambiente para a colaboração, os requisitos da coordenação possui como objetivo: gerir os participantes nas atividades colaborativas. Devido as interdependências entre tarefas na colaboração alguns conflitos podem ocorrer, em razão disso a cooperação nem sempre é harmoniosa. Atividades envolvendo múltiplos indivíduos exigem um planejamento formal, e muitas vezes as atividades ligadas com as relações sociais, ou com trabalho colaborativo, devem ser bem controladas com mecanismos de coordenação explícitos.

Os requisitos de coordenação, na maioria dos casos, são refinamentos das regras estabelecidas na fase de articulação. Assim, novos compromissos são assumidos e/ou renegociados. Prazos e permissões podem ser redefinidos para atender os objetivos do trabalho colaborativo. Os Papéis também podem sofrer alterações, quando múltiplos perfis estão em um mesmo espaço compartilhado.

Em especial, existe um requisito de coordenação chamado de Multiplicidade de Papéis que possui a responsabilidade de suportar a delegação de mais de um perfil a um mesmo usuário, dentro de um mesmo grupo de colaboradores. Este requisito está contido em atividades mais complexas e necessita de gestão de permissões alinhavadas à sua complexidade de aplicação. Podemos perceber a utilização deste requisito, quando temos duas ou mais instâncias de uma AP para um mesmo grupo de usuários (ex: Grupo Debate). Onde temos o usuário A com o perfil de Argumentador em subgrupo X e o mesmo usuário com o perfil de Revisor no subgrupo Y. O mecanismo de coordenação deverá gerenciar as permissões em cada instância de aplicação para torná-las consistentes em seus respectivos subgrupos. 


\section{Avaliação e Resultados}

Após o levantamento dos requisitos de articulação e coordenação realizamos a experimentação da implementação de cada AP nos AVAs selecionados. A tabela 3 exibe uma compilação dos resultados extraídos do experimento.

Definimos uma nota qualitativa a ser atribuída a cada ambiente, segundo a conformidade com cada requisito, que foram: [3] - atende plenamente com a descrição do requisito; [2] - atende parcialmente e requer pequenas adaptações; [1] - atende parcialmente e requer muitas adaptações; [0] - não há recursos que suportem o requisito.

Tabela 2: Avaliação do suporte dos requisitos nos AVAs.

\begin{tabular}{|c|c|c|c|c|c|c|c|c|c|}
\hline & Requisito/AVA & Moodle & ATutor & Caroline & Amadeus & Sócrates & Chamilo & Dokeos & Eureka \\
\hline \multirow{10}{*}{ 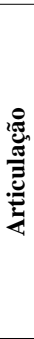 } & C.E.R. & 3 & 3 & 3 & 3 & 3 & 3 & 3 & 3 \\
\hline & Convidar usuários & 3 & 3 & 3 & 2 & 3 & 3 & 3 & 3 \\
\hline & Determinação de Papeis & 3 & 3 & 3 & 1 & 2 & 3 & 2 & 3 \\
\hline & Dependência de Atividades & 2 & $\mathbf{1}$ & 1 & 1 & 1 & 1 & 1 & 1 \\
\hline & Restrição de Interações* & $\mathbf{0}$ & $\mathbf{0}$ & $\mathbf{0}$ & 1 & 1 & $\mathbf{0}$ & $\mathbf{0}$ & $\mathbf{0}$ \\
\hline & Perfis Dinâmicos*** & $\mathbf{0}$ & $\mathbf{0}$ & $\mathbf{0}$ & $\mathbf{0}$ & $\mathbf{0}$ & $\mathbf{0}$ & $\mathbf{0}$ & $\mathbf{0}$ \\
\hline & Definir Permissão de Leitura & 1 & 1 & $\mathbf{0}$ & $\mathbf{0}$ & $\mathbf{0}$ & 1 & 1 & $\mathbf{0}$ \\
\hline & \begin{tabular}{|l|} 
Definir Prazo de Leitura \\
\end{tabular} & $\mathbf{1}$ & $\mathbf{0}$ & 1 & 1 & 1 & 1 & 1 & $\mathbf{1}$ \\
\hline & Definir Permissão de Escrita & 1 & 1 & $\mathbf{0}$ & $\mathbf{0}$ & $\mathbf{0}$ & 1 & 1 & $\mathbf{0}$ \\
\hline & \begin{tabular}{|l|} 
Definir Prazo de Escrita \\
\end{tabular} & $\mathbf{1}$ & 1 & 1 & 1 & 1 & 1 & 1 & $\mathbf{1}$ \\
\hline \multirow{5}{*}{ 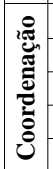 } & \begin{tabular}{|l|} 
Multiplicidade de Papeis \\
\end{tabular} & 1 & $\mathbf{0}$ & $\mathbf{0}$ & $\mathbf{0}$ & 1 & $\mathbf{0}$ & $\mathbf{0}$ & 1 \\
\hline & Redefinir Permisão de Leitura & $\mathbf{1}$ & $\mathbf{1}$ & $\mathbf{0}$ & $\mathbf{0}$ & $\mathbf{0}$ & 1 & 1 & $\mathbf{0}$ \\
\hline & \begin{tabular}{|l|} 
Redefinir Prazo de Leitura \\
\end{tabular} & 1 & $\mathbf{0}$ & $\mathbf{0}$ & 1 & 1 & 1 & 1 & 1 \\
\hline & \begin{tabular}{|l|} 
Redefinir Permissão de Escrita \\
\end{tabular} & 1 & 1 & $\mathbf{0}$ & $\mathbf{0}$ & $\mathbf{0}$ & 1 & 1 & $\mathbf{0}$ \\
\hline & Redefinir Prazo de Escrita & 1 & 1 & $\mathbf{0}$ & 1 & 1 & 1 & 1 & 1 \\
\hline
\end{tabular}

Observamos que os AVAs avaliados não se mostraram adequados para suportar um protocolo de interação mais complexo, como os que exemplificamos a partir de algumas arquiteturas pedagógicas. Em outras palavras, esperar dos AVAs uma flexibilidade para apoiar práticas pedagógicas, que requerem uma alta complexidade de interações é exigir algo além das atividades para as quais foram projetados. Percebemos que a arquitetura de softwares dos AVAs está voltada para a disponibilização de ferramentas específicas (fórum, wiki, chat...) com propósitos definidos e limitados, o que explica o fato destas ferramentas não oferecerem recursos passíveis de configuração.

Considerando que as ferramentas não oferecem nenhum suporte para a gestão da coordenação da interação, todo o processo de implementação das APs necessita de muitas adaptações para viabilizar, ainda que parcialmente, que uma estratégia pedagógica seja implementada com razoável eficácia, quando possível.

De nossa avaliação, pudemos concluir que enquanto as ferramentas disponíveis nos AVAs não avançarem em torno da flexibilidade, principalmente na gestão de Permissões e Papéis, estes ambientes não se mostram adequados para dar suporte a propostas mais complexas para a cooperação.

O Moodle e o Chamilo, que apesar de possuírem algum destaque comparado aos demais, ainda estão muito longe do que é necessário para suportar os requisitos na implementação das APs. Para exemplificar, uma Instância da AP construindo conceituações possuiu um suporte de implementação abaixo dos $40 \%$ dos requisitos e mesmo quando suportado as adaptações eram de grande esforço. $O$ esforço nas adaptações ocorre, tanto nos aspectos administrativos (como administrador), quanto nos aspectos de participação (como colaborador).

O resultado da avaliação realizada corrobora a hipótese de que atualmente ainda temos uma grande lacuna tecnológica para preenchermos no tocante ao uso de ambientes 
virtuais para apoio à aprendizagem cooperativa e que se faz necessário a concepção de ambientes com características flexíveis para a modelagem de interações complexas. Enquanto isto não ocorrer, a realização de novas propostas pedagógicas implicará no desenvolvimento de softwares específicos ou, o que é mais provável de acontecer, as novas propostas continuarão a serem podadas pelas limitações dos ambientes.

\section{Conclusão}

Segundo Carvalho et al. (2005),

Utilizar APs pressupõe equilibrar componentes fundamentais: concepção pedagógica forte, sistematização metodológica e suporte telemático. A confluência dessas perspectivas é que permitem aos estudantes disporem de atividades cognitivamente instigantes e desenvolver métodos de trabalho interativos e construtivos. (Carvalho et al., 2005).

Com o propósito de avaliar o estado da arte com respeito às reais possibilidades de como AVAs oferecem suporte computacional à propostas pedagógicas com interações complexas, procedemos um estudo onde identificamos características de coordenação requeridas por algumas APs. De posse desses requisitos analisamos as possibilidades oferecidas para descrição dessas características em alguns ambientes conhecidos. Identificamos com isso uma lacuna nas abordagens correntes de AVAs com respeito aos objetivos de oferecer apoio computacional à novas propostas pedagógicas.

Com isto, pudemos evidenciar que os AVAs possuem um suporte telemático capaz de satisfazer os cursos online e sua metodologia de ensino. Entretanto, o que podemos constatar é uma frequiente adaptação nas propostas pedagógicas aos ambientes virtuais e não o contrário, como se deseja. Esta percepção é fortalecida quando visualizamos a falta de flexibilidade na gestão do trabalho colaborativo, apresentado neste trabalho. Reduzindo a capacidade de implementar fortes concepções pedagógicas em espaços virtuais com diretrizes voltada à aprendizagem.

Os Requisitos levantados nesse estudo são críticos para o uso dos AVAs no suporte à propostas pedagógicas de interação complexa e podem guiar a elaboração de novas propostas para esses ambientes. Acreditamos que estes requisitos não estão apenas associados aos LMS, mas também a outros groupwares com finalidades distintas, porém associados a alta necessidades de gestão da colaboração.

\section{Referências Bibliográficas}

BORGHOFF, U.M.; SCHLICHTER, J.H. Computer supported cooperative work: Introduction to distributed applications. Berlin: Springer-Verlag. 2000.

CARVALHO, M.J.S.; NEVADO, R.A., MENEZES, C.S. Arquiteturas Pedagógicas para Educação a Distância: Concepções e Suporte Telemático, SBIE, Juiz de Fora - MG, 2005.

ELLIS, C.A.; Simon J.G.; Gail R. Groupware: some issues and experiences, Communications of the ACM, v.34 n.1, p.39-58, Jan. 1991

EUREKA, Site Oficial do Ambiente Virtual Eureka. Disponível em <https://eureka.pucpr.br/>. Acesso em: 16 maio. 2011.

FAGUNDES, L., NEVADO, R., BASSO, M., BITENCOURT, J., MENEZES, C., MONTEIRO, V.C.P.C. “ Projetos de Aprendizagem - Uma experiência mediada por ambientes Telemáticos”. Revista Brasileira de Informática na Educação. 2006.

DOKEOS, Site Oficial. Disponível em <http://dokeos.com>. Acesso em: 15 maio 2011 
FUKS, H.; RAPOSO, A.; GEROSA, M.A.; PIMENTEL, M.; LUCENA, C.J.P. The 3C Collaboration Model. The Encyclopedia of E-Collaboration, Ned Kock (org), ISBN 978-1-59904-000-4, pp. 637-644, 2007.

GOMES, A. S.; TEDESCO, P. A. "Amadeus: A Framework To Constructivist Support Based on Projects and MultiDimensional Learner Evaluation". In: ELearn 2002World Conference on ELearning in Corporate, Government, Healthcare, and Higher Education, Montreal. Proceedings of Elearn. 2002.

JOHNSON, D.W., JOHNSON, R., \& SMITH, K. "Structuring Academic Controversy". In: Sharan, Shlomo. Handbook of Cooperative Learning Methods. Praeger Publishers. London, 1994.

LENGYEL, P.; HERDON, M.; SZILÁGYI, R. Comparison of Moodle and ATutor LMSs. University of Debrecen Centre of Agricultural Sciences, Faculty of Agricultural Economics and Rural Development, 2006.

LIU, J.; MEN, H.; HAN, J. Comparative Study of Open-source E-learning Management Platform. Computational Intelligence and Software Engineering, 2009. CiSE 2009. International Conference on , vol., no., pp.1-4, 11-13 Dec. 2009

MAES, J.M.; Chamilo 2.0: A Second Generation Open Source E-learning and Collaboration Platform. University College Ghent, Ghent, Belgium. 2010.

MENEZES, C.; NEVADO, R.; CASTRO Jr., A.; SANTOS, L. MOrFEU - MultiOrganizador Flexível de Espaços VirtUais para Apoiar a Inovação Pedagógica em EAD. Anais do XIX Simpósio Brasileiro de Informática na Educação. Fortaleza-CE, 2008.

MOODLE, Moodle.org: open-source community- based tools for learning. Disponível em <http://moodle.org/>. Acesso em: 15 maio. 2011.

MONTEIRO, V. C. P. C.; MENEZES, C. S.; NEVADO, R. A.; FAGUNDES, L. C. Ferramenta de Autoria e Interação para apoio ao desenvolvimento de Projetos de Aprendizagem. Renote Revista Novas Tecnologias na Educação V3, v. 3, n. 2, 2005.

NASCIMENTO, M. S. P.; SILVA, M. A.; VIANA JUNIOR, G.; ROCHA, B. T. S.; VENTURA, P. P. B.; PAULA, D. F.; CASTRO FILHO, J. A. "SÓCRATES - Sistema Colaborativo de Apoio à Educação On-line.”. XIX Simpósio Brasileiro de Informática Educativa - SBIE, 2008, Fortaleza-CE. 2008.

NEVADO, R.A. ; DALPIAZ, M.M. ; MENEZES, C.S. . Arquitetura Pedagógica para Construção Colaborativa de Conceituações. In: Xv Workshop sobre Informática na Escola, 2009, Bento Gonçalves - RS. 2009. v. 1.

PESSOA, J. M.; MENEZES, C. S. Framework Baseado em Padrões Abertos para Construção de Ambientes CSCW/CSCL na Web, in Proceedings of the First Latin American Web Congress -La-Web. 0-7695-2058-8/03 - 2003 IEEE. 2003.

REAL, L. M. C., MENEZES, C. S. Júri simulado: possibilidade de construção de conhecimento a partir de interações em grupo. Aprendizagem em rede na educação a distância: estudos e recursos para formação de professores. Porto Alegre. 2007.

SCHMIDT, K.; BANNON, L.J. Taking CSCW seriously - Supporting articulation work. Computer Supported Cooperative Work, 1(2), Kluwer, p 7-40. 1992.

SERRES, F. F., BASSO, M. V. A. "Diários virtuais - Uma ferramenta de comunicação social para a autoria e aprendizagem de Matemática", Anais do Simpósio Brasileiro de Informática na Educação. 2009. 